\title{
Effects of eplerenone, a selective mineralocorticoid receptor antagonist, on clinical and experimental salt-sensitive hypertension
}

\author{
Yoshiyu Takeda
}

Mineralocorticoid receptors (MRs) are expressed in non-epithelial tissues, such as blood vessels, the heart and adipose tissue. The combined effects of aldosterone and insulin link the metabolic syndrome with hypertension and salt sensitivity. Eplerenone is the newly developed inhibitor of MRs that has significantly fewer adverse effects than similar doses of spironolactone. Eplerenone has been reported to have anti-hypertensive and protective effects on cardiovascular and renal injury in salt-sensitive hypertensive animal models, such as the Dahl salt-sensitive (DS) hypertensive rat and leptin receptor-deficient spontaneously hypertensive rat (SHR/cp). Eplerenone also increases nitric oxide bioavailability and improves impaired endothelial function by decreasing oxidative stress. Clinical studies support the concept that eplerenone is effective for the treatment of salt-sensitive hypertension as well as idiopathic hyperaldosteronism and does not have adverse anti-androgenic adverse effects. In Japan, eplerenone has been used clinically since $\mathbf{2 0 0 7}$ for the treatment of hypertension, with its price being marginally lower than all types of angiotensin II receptor antagonists. This will inevitably result in an increasing number of hypertensive patients and those with primary aldosteronism being treated with this agent in the near future.

Hypertension Research (2009) 32, 321-324; doi:10.1038/hr.2009.29; published online 20 March 2009

Keywords: aldosterone; eplerenone; obese; renin; salt

\section{INTRODUCTION}

Aldosterone plays an important role in the pathogenesis of cardiovascular, renal and metabolic diseases. Mineralocorticoid receptors (MRs) exist in both epithelial and non-epithelial cells, such as vascular endothelial and smooth muscle cells, cardiomyocytes and adipocytes. Experimental studies have shown that the profibrotic actions of aldosterone develop only in association with a high-sodium diet. Excess sodium intake is a major contributing factor in the pathogenesis of hypertension, and large population-based studies have shown that there is a significant correlation between the level of salt intake and blood pressure and the frequency of hypertension. In this report, we review the clinical and experimental evidence for the use of the selective receptor antagonist, eplerenone, in the treatment of saltsensitive hypertension. This review also examines how this evidence has contributed to our understanding of the pathophysiological mechanisms of salt-sensitive hypertension.

\section{SALT, ALDOSTERONE AND HYPERTENSION}

Recent studies including our own have shown that $\sim 10 \%$ of patients with essential hypertension have a high ratio of plasma aldosterone concentration (PAC)/plasma renin activity (PRA), suggesting an inappropriate increase in aldosterone synthesis. Makhanova et al. ${ }^{1}$ reported that transgenic mice with increased expression of aldosterone synthase (AS) developed salt-sensitive hypertension and cardiac hypertrophy associated with increased oxidative stress. On the basis of these findings, it was suggested that genetically determined increases in the AS expression in humans may contribute to hypertension and cardiovascular complications in societies on high-salt diets.

Aldosterone is involved in vascular smooth muscle hypertrophy and can cause vascular matrix impairment and endothelial dysfunction. Tobian and Redleaf ${ }^{2}$ proposed that aldosterone influences salt and water balance in vascular smooth muscle cells (VSMCs), thereby affecting vessel lumen size. Kornel et al. ${ }^{3}$ presented evidence that glucocorticoids and mineralocorticoids controlled the contractility of VSMC, with this control being mediated by glucocorticoid and MRs on these cells. Farquharson and Struthers ${ }^{4}$ reported that spironolactone induced marked increases in endothelial nitric oxide bioactivity and that the magnitude of this increase (94\%) was considerably greater than that in other therapies, such as angiotensin-converting enzyme (ACE) inhibitors and statins, which usually improved the endothelial dysfunction by only 25-35\%. Improving endothelial dysfunction is considered to be important, as such improvements 
are likely to be associated with a reduced incidence of future cardiovascular events. Nishizaka et al. ${ }^{5}$ reported a strong association in humans between aldosterone excess and impaired endothelial function, measured by flow-mediated arterial vasodilatation. They also showed that high-salt intake induced further deleterious effects on renal function in hypertensive patients with hyperaldosteronism compared with those with essential hypertension.

Aldosterone also exerts direct profibrotic effects. Studies of Brilla et al. ${ }^{6}$ showed that myocardial fibrosis developed after a prolonged period of exposure to aldosterone in the presence of a moderate level of salt intake. Similarly, Rocha et al. ${ }^{7}$ reported that the aldosterone/salt treatment induced coronary inflammation, characterized by monocyte and macrophage infiltration and by increased expression of the inflammatory markers, cyclooxygenase-2, osteopontin, macrophage chemoattractant protein-1 and intracellular adhesion molecule-1. Eplerenone was found to partially decrease blood pressure and attenuate the inflammatory changes in this experimental model. These findings suggest that the cardiac fibrosis induced by aldosterone in a high-salt diet is a secondary event in response to vascular cytokine activation, inflammatory damage and necrotic changes. Kagiyama et al. ${ }^{8}$ reported that aldosterone- and salt-induced cardiac fibrosis is independent from angiotensin II type 1a receptor (ATlaR) signaling, using AT1R knockout mice. The cardioprotective effects of eplerenone are blood pressure-independent in mice that lack the gene encoding guanylyl cyclase-A. ${ }^{9}$ Urabe et al. ${ }^{10}$ also showed that eplerenone improved the left ventricular remodeling after myocardial infarction in rats that were fed a high-salt diet but not in those fed a low-salt diet.

\section{INTER-RELATIONSHIPS BETWEEN THE METABOLIC SYNDROME, SALT AND ALDOSTERONE}

The metabolic syndrome, characterized by a clustering of glucose intolerance, dyslipidemia, central obesity and hypertension, is a strong predictor of cardiovascular and renal disease. There is increasing evidence that aldosterone and salt have pathophysiological roles in the metabolic syndrome. Ehrhart-Bornstein et al. ${ }^{11}$ showed that human adipocytes secrete aldosterone-releasing factors and suggested a direct link between obesity and hypertension. The SHR rat has been used extensively in studies of hypertension, whereas a leptin receptordeficient SHR (SHR/cp) has been developed as a model of the human metabolic syndrome. Nagase et al. ${ }^{12}$ reported that eplerenone blocked renal damage caused by an intake of a high-salt diet in these SHR/cp rats. Recent studies have suggested that aldosterone and MRs may be involved in adipocyte biology, given the high binding affinity of these receptors for both mineralocorticoids and glucocorticoids. There is also evidence from studies of Caprio et al. ${ }^{13}$ that aldosterone promotes the maturation of pre-adipocytes to adipocytes cells in a time-, doseand MR-dependent manner. Aldosterone plasma levels are elevated in obesity and in patients with the metabolic syndrome. Bentley-Lewis et al. ${ }^{14}$ showed that aldosterone production was elevated and associated with insulin resistance in overweight adults on a high-sodium diet. The National Nutrition Survey in Japan showed a high-sodium intake in recent years compared with that in Western countries. Ohta et al. ${ }^{15}$ reported that urinary salt excretion was higher in hypertensive patients with the metabolic syndrome than that in those without the metabolic syndrome. Further studies are needed to examine the potential benefit of MR blockade on insulin resistance in patients with the metabolic syndrome.

\section{MINERALOCORTICOID-STIMULATING FACTORS}

Under physiological conditions, the main stimuli for adrenal zona glomerulosa-derived aldosterone secretion are angiotensin II (Ang II), potassium and adrenocorticotropin. Kidambi et al. ${ }^{16}$ showed that PAC correlated positively with blood pressure, waist circumference and insulin resistance index in patients with hypertension and the metabolic syndrome, whereas plasma potassium levels and PRA were similar in patients with the metabolic syndrome and in those without. Bentley-Lewis et al. ${ }^{14}$ reported that urinary aldosterone excretion and Ang II-stimulated aldosterone were increased in overweight patients compared with that in lean, normotensive patients, although PRA, urinary cortisol excretion and plasma potassium levels did not differ between the two groups. These observations suggest that aldosterone synthesis is controlled by an as-yet-unknown factor(s) in patients with the metabolic syndrome. Recently, Ehrhart-Bornstein et al. ${ }^{11}$ suggested an excellent theory that human adipocytes secrete mineralocorticoidstimulating factors, which control the production of aldosterone by the adrenal gland. Using in vitro studies, Nagase et al. ${ }^{12}$ showed that visceral adipocytes from obese animals with the metabolic syndrome secreted factors that enhanced aldosterone secretion from adrenocortical cells, whereas this effect was not observed in lean control animals. The original report by Ehrhart-Bornstein et al. ${ }^{11}$ clearly shows that these so-called mineralocorticoid-stimulating factors stimulated not only aldosterone but also cortisol. Glucocorticoid is a key hormone in Cushing's syndrome, a disorder that is characterized by obesity, hypertension and glucose intolerance resembling a severe form of the metabolic syndrome. The importance of local effects of glucocorticoids has also been emphasized in studies on the action of $11 \beta$ hydroxysteroid dehydrogenase 1 (11 $\beta$-HSD1), an enzyme that catalyses the formation of the active hormone cortisol (corticosterone in rodents) from its less active precursor, cortisone (11-dehydrocorticosterone in rodents). Transgenic mice that overexpress 11ß-HSD1 in adipocytes develop obesity, insulin resistance, dyslipidemia, salt-sensitive hypertension and activation of the systemic renin-angiotensinaldosterone system (RAAS). ${ }^{17}$ Taken together, these findings suggest the existence of mineralocorticoid-stimulating factors, although identifying these factors is urgently required for confirming this interesting concept.

\section{EFFECTS OF EPLERENONE IN EXPERIMENTAL ANIMAL MODELS WITH SALT-SENSITIVE HYPERTENSION}

Dahl salt-sensitive (DS) rats are widely used to study the pathophysiology of salt-sensitive hypertension. In this strain, supplemental dietary sodium increases blood pressure, whereas in the Dahl saltresistant (DR) strain, it has little or no effect on blood pressure. There are several reports of the abnormalities of the renin-angiotensin system, adrenal steroids and sympathetic nerve system in the DS rats. There has been increasing evidence that mineralocorticoids, acting directly on peripheral vascular tissues, cause hypertension. MR binding is tightly regulated by the enzyme $11 \beta$-hydroxysteroid dehydrogenase 2 (11 $\beta$-HSD2), which selectively metabolizes glucocorticoids to inactive metabolites, thus allowing for MR activation by aldosterone. We have reported earlier that vascular 11 $\beta$-HSD2 activity was decreased in the DS rats compared with that in DR rats. ${ }^{18}$ We found that treatment with eplerenone in the DS rats not only decreased blood pressure but also improved tissue injuries. ${ }^{19}$ Nagase et $a .^{20}$ reported the same results in the DS rats. Endemann et al. ${ }^{21}$ showed that eplerenone decreased blood pressure only in stroke-prone spontaneously hypertensive rats (SHRSP) fed a high-sodium diet, but not in those fed a low-sodium diet. Why does MR blockade decreases blood pressure in spite of decreased circulating RAAS in salt-sensitive hypertensive rats? Kobori et al. ${ }^{22}$ reported that a high-sodium diet decreased circulating RAS in both the DS and the DR rats. However, intrarenal angiotensinogen was enhanced in the DS rats but not in the 


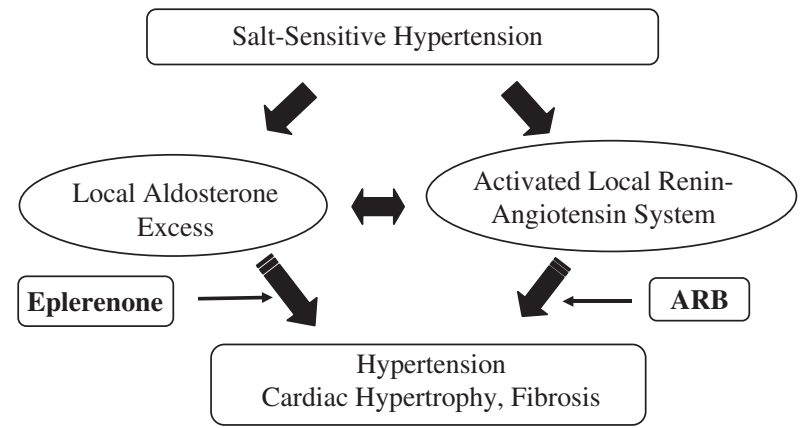

Figure 1 High-sodium diet activates the local renin-angiotensin-aldosterone system (RAAS) in cardiovascular tissues, which caused hypertension and cardiac hypertrophy in salt-sensitive hypertension. Treatment with eplerenone and/or angiotensin II type 1 receptor (AT1R) blocker prevents hypertension, cardiac hypertrophy and fibrosis and improves oxidative stress.

DR rats, suggesting that paradoxical responses of tissue RAAS by a high-sodium diet may contribute to the development of hypertension. In our experimental data, ${ }^{23}$ tissue angiotensinogen and ACE gene expression were increased in the DS rats. Jaffe and Mendelsohn ${ }^{24}$ reported that Ang II increased vascular MR gene expression. Taken together, a high-sodium diet increases tissue Ang II, which activates local MR in the DS rats and thus, increased local RAAS may be an important contributing factor to the progression of hypertension, cardiac hypertrophy and fibrosis in salt-sensitive hypertension (Figure 1). Recently, Fujita's ${ }^{25}$ group has proposed aldosterone-independent MR activation in salt-sensitive hypertension. They have identified a new role for Racl, a member of the Rho family of GTPases, as a potent activator of MR signal transduction both in vitro and in vivo. ${ }^{26}$ The constitutively active Racl enhances nuclear translocation and the transcriptional activity of MR in vitro. They showed that a unique Rac-specific inhibitor, NSC23766, effectively ameliorates proteinuria and glomerular damage in the DS rats.

\section{EFFECTS OF EPLERENONE IN PATIENTS WITH SALT-SENSITIVE HYPERTENSION}

Clinical studies support the concept that eplerenone is effective for the treatment of hypertension without showing adverse anti-androgenic adverse effects. Patients with hypertension and low renin activity, a combination that indicates a suppression of the RAAS, are often saltsensitive, and RAS inhibitors, such as ACE inhibitors and angiotensin receptor blockers (ARBs) may not be particularly effective in this population that includes a high prevalence of black patients. This subgroup of patients may respond better to eplerenone, which reduces sodium and fluid retention by the kidneys. ${ }^{27}$ However, a comparison with thiazide diuretics, which can also be effective in these patients, is currently lacking. There are, however, considerable clinical data that treatment with thiazide diuretics in patients with hypertension induces glucose intolerance or diabetes mellitus concomitantly with hypokalemia. ${ }^{28}$ When the central role of insulin resistance in the metabolic syndrome is taken into account, it is possible that the syndrome is linked to salt-sensitive hypertension. Fujita ${ }^{29}$ reported an association between the presence of the metabolic syndrome and saltsensitive hypertension. Aldosterone bound to MRs binds to the serum and glucocorticoid regulated 1 (SGK1) promoter, resulting in increased SGK1 expression, whereas insulin, through its effects on phosphatidylinositol-3-kinase, activates SGK1 through phosphorylation. This activation of SGK1 by insulin, in combination with increased SGK1 expression by aldosterone, inhibits the protein neu- ronal precursor cell, expressed developmentally after the downregulation of 4-like (NEDD4L). These changes, in turn, result in the removal of amiloride-sensitive epithelial sodium channels $(\mathrm{ENaC})$ from renal tubular cells, the expression of which, causes increased renal sodium re-absorption and salt sensitivity. ${ }^{30}$ Taken together, eplerenone may, therefore, be more effective for the treatment of salt-sensitive hypertension than thiazide diuretics.

\section{EFFECTS OF EPLERENONE IN PATIENTS WITH PRIMARY ALDOSTERONISM}

Primary aldosteronism represents the most common form of secondary hypertension. It has been reported that $5-13 \%$ of unselected hypertensive patients have primary aldosteronism. We reported that these patients have a higher incidence of cardiovascular complications than age- and sex-matched patients with essential hypertension. ${ }^{31}$ Idiopathic hyperaldosteronism (IHA) is the most common subtype of primary aldosteronism and accounts for $40-60 \%$ of cases. Karagiannis et al..$^{32}$ showed that treatment with eplerenone was more effective than with sprironolactone for lowering blood pressure and improving hypokalemia in patients with IHA. Treatment with eplerenone also reduced the frequency of sex-hormone-related adverse effects compared with that with spironolactone. In that report, two patients who received spironolactone developed painful bilateral gynecomastia that was completely resolved when they were switched to eplerenone. No cases of gynecomastia were observed in patients treated with eplerenone.

\section{EPLERENONE HAS A FUTURE IN THE MANAGEMENT OF HYPERTENSION IN JAPAN}

As mentioned above, eplerenone may be effective for the treatment of salt-sensitive hypertension, the metabolic syndrome and IHA. This agent also has many organ-protective effects independent of its blood pressure-lowering effect and fewer side effects, with the exception of diabetic patients with renal insufficiency. In Europe, Australia and the United States, the price of eplerenone is more than twice that of ARBs. ${ }^{33}$ In Japan, eplerenone has been used clinically since 2007 for the treatment of hypertension with the price of the 50-mg dose being marginally lower than all types of ARBs. As a consequence, it would be anticipated there will be an increasing number of patients with hypertension, including those with primary aldosteronism who will be treated with eplerenone in the near future.

\section{CONFLICT OF INTEREST}

The authors declare no conflict of interest.

1 Makhanova N, Hagaman J, Kim H-S, Smithies O. Salt-sensitive blood pressure in mice with increased expression of aldosterone synthase. Hypertension 2008; 51: 134-140.

2 Tobian L, Redleaf PD. Effect of hypertension on arterial wall electrolytes during deoxycorticosterone administration. Am J Physiol 1957; 189: 451-454.

3 Kornel L, Rafelson ME, Hayashi T, Kanamarlapudi N, Anderson KM. Receptors for adrenal steroids and transport of electrolytes in vascular smooth muscle. Clin Physiol Biochem 1988; 6: 188-200.

4 Farquharson C, Struthers AD. Spironolactone increases NO bioactivity, improves endothelial dysfunction and suppresses vascular Al/All conversion in chronic heart failure. Circulation 2000; 101: 594-597.

5 Nishizaka M, Zaman MA, Sharon A, Green SA, Renfroe KY, Calhoun DA. Impaired endothelium-dependent flow-mediated vasodilation in hypertensive subjects with hyperaldosteronism. Circulation 2004; 109: 2857-2861.

6 Brilla CG, Pick R, Tan LB, Janicki JS, Weber KT. Remodeling of the right and left ventricles in experimental hypertension. Circ Res 1990; 67: 1355-1364.

7 Rocha R, Martin-Berger CL, Yang P, Scherrer R, Delyani J, McMahon E. Selective aldosterone blockade prevents angiotensin II/salt-induced vascular inflammation in the rat heart. Endocrinology 2002; 143: 4828-4836. 
8 Kagiyama S, Matsumura K, Fukuhara M, Sakagami K, Fujii K, lida M. Aldosterone-andsalt-induced cardiac fibrosis is independent from angiotensin II type 1a receptor signaling in mice. Hypertens Res 2007; 30: 979-989.

9 Zhang Q, Saito Y, Naya N, Imagawa K, Somekawa S, Kawata H, Takeda Y, Uemura S, Kishimoto I, Nakao K. The specific mineralocorticoid receptor blocker eplerenone attenuates left ventricular remodeling in mice lacking the gene encoding guanylyl cyclase-A. Hypertens Res 2008; 31: 1251-1256.

10 Urabe A, Izumi T, Abe Y, Taniguchi I, Mochizuki S. Effects of eplerenone and salt intake on left ventricular remodeling after myocardial infarction in rats. Hypertens Res 2006; 29: 627-634.

11 Ehrhart-Bornstein M, Lamounier-Zepter V, Schraven A, Langenbach J, Willenberg HS, Barthel A, Hauner H, McCann SM, Scherbaum WA, Bornstein SR. Human adipocytes secrete mineralocorticoid-releasing factors. Proc Natl Acad Sci USA 2003; 100: 14211-14216.

12 Nagase M, Matsui H, Shibata S, Gotoda T, Fujita T. Salt-induced nephropathy in obese spontaneously hypertensive rats via paradoxical activation of the mineralocorticoid receptor, role of oxidative stress. Hypertension 2007; 50: 877-883.

13 Caprio M, Fève $B$, Claës $A$, Viengchareun S, Lombès $M$, Zennaro M-C. Pivotal role of the mineralocorticoid receptor in corticosteroid-induced adipogenesis. FASEB J 2007; 21 : 2185-2194.

14 Bentley-Lewis R, Adler GK, Perlstein T, Seely EW, Hopkins PN, Williams GH, Garg R. Body mass index predicts aldosterone production in normotensive adults on a high-salt diet. J Clin Endocrinol Metab 2007; 92: 4472-4475.

15 Ohta Y, Tsuchihashi T, Arakawa K, Onaka U, Ueno M. Prevalence and lifestyle characteristics of hypertensive patients with metabolic syndrome followed at an outpatient clinic in Fukuoka, Japan. Hypertens Res 2007; 30: 1077-1082.

16 Kidambi S, Kotchen JM, Grim CE, Raff H, Mao J, Singh RJ, Kotchen TA. Association of adrenal steroids with hypertension and the metabolic syndrome in blacks. Hypertension 2007; 49 (Part 2): 704-711.

17 Masuzaki H, Yamamoto H, Christopher J, Kenyon CJ, Elmquist JK, Morton NM, Paterson JM, Shinyama H, Sharp MGF, Fleming S, Mullins JJ, Seckl JR, Jeffrey S, Flieret JS. Transgenic amplification of glucocorticoid action in adipose tissue causes high blood pressure in mice. J Clin Invest 2003; 112: 83-90.

18 Takeda Y, Inaba S, Furukawa K, Miyamori I. Renal 11ß-hydroxysteroid dehydrogenase in genetically salt-sensitive hypertensive rats. Hypertension 1998; 32: 1077-1082.

19 Takeda Y, Zhu A, Yoneda T, Usukura M, Takata H, Yamagishi M. Effects of aldosterone and angiotensin II receptor blockade on cardiac angiotensinogen and angiotensin- converting enzyme 2 expression in Dahl salt-sensitive hypertensive rats. Am J Hytertens 2007; 20: 1119-1124.

20 Nagase M, Shibata S, Yoshida S, Nagase T, Gotoda T, Fujita T. Podocyte injury underlies the glomerulopathy of Dahl salt-hypertensive rats and is reversed by aldosterone blocker. Hypertension 2006; 47: 1084-1093.

21 Endemann DH, Touyz RM, Iglarz M, Savoia C, Schiffrin E. Eplerenone prevents saltinduced vascular remodeling and cardiac fibrosis in stroke-prone spontaneously hypertensive rats. Hypertension 2004; 43: 1252-1257.

22 Kobori H, Nishiyama A, Abe Y, Navar LG. Enhancement of intrarenal angiotensinogen in Dahl salt-sensitive rats on high salt diet. Hypertension 2003; 41: 592-597.

23 Zhu A, Yoneda T, Demura M, Karashima S, Usukura M, Yagi K, Yamagishi M, Takeda Y. Effect of mineralocorticoid receptor blockade on the renal renin-angiotensin-system in Dahl salt-sensitive hypertensive rats. J Hypertens 2009 (In press).

24 Jaffe IZ, Mendelsohn ME. Angiotensin II and aldosterone regulate gene transcription via functional mineralocorticoid receptors in human coronary artery smooth muscle cells. Circ Res 2005; 96: 643-650.

25 Fujita T. Aldosterone in salt-sensitive hypertension and metabolic syndrome. J Mol Med 2008; 86: 729-734.

26 Shibata S, Nagase M, Yoshida S, Kawarazaki W, Kurihara H, Tanaka H, Miyoshi J, Takai Y, Fujita T. Modification of mineralocorticoid receptor function by Rac1 GTPase: implication in proteinuric kidney disease. Nature Med 2008; 14: 1370-1376.

27 Flack JM, Oparil S, Pratt JH, Roniker B, Garthwaite S, Kleiman JH, Yang Y, Krause SL, Workman D, Saunders E. Efficacy and tolerability of eplerenone and losartan in black and white patients. Am J Coll Cardiol 2003; 41: 1148-1155.

28 Zillich AJ, Garg J, Basu S, Bakins GL, Carter BL. Thiazide diuretics, potassium, and the development of diabetes: a quantitative review. Hypertension 2006; 48: 219-224.

29 Fujita T. Insulin resistance and salt-sensitive hypertension in metabolic syndrome. Nephrol Dial Transplant 2007; 11: 3102-3107.

30 Kamynina E, Staub O. Concerted action of ENaC, Nedd4-2, and Sgk1 in transepithelial $\mathrm{Na}(+)$ transport. Am J Physiol Renal Physiol 2002; 283: F377-F387.

31 Takeda Y. Genetic alterations in patients with primary aldosteronism. Hypertens Res $2001 ; 24: 469-474$

32 Karagiannis A, Triomalos K, Papageorgiou A, Kakafika AI, Pagourelias ED, Anagnostis P, Athyros VG, Mikhailidis DP. Spironolactone versus eplerenone for the treatment of idiopathic hyperaldosteronism. Expert Opin Pharmacother 2008; 9: 509-515.

33 Gosse P, Macfadyen RJ. Does eplerenone have a future in the management of hypertension in Europe? J Human Hypertens 2006; 20: 829-832. 